https://doi.org/10.15407/ujpe63.6.495

A.V. TYKHYY

Institute for Nuclear Research, Nat. Acad. of Sci. of Ukraine

(47, Prosp. Nauky, Kyiv 03680, Ukraine; e-mail: tykhyy@kinr.kiev.ua)

\title{
STOCHASTIC DIFFUSION OF ENERGETIC IONS IN WENDELSTEIN-TYPE STELLARATORS
}

\begin{abstract}
The collisionless stochastic diffusion of energetic ions in optimized stellarators of the Wendelstein type has been considered. The phenomenon concerned was predicted earlier in the framework of a simplified theory describing the separatrix crossing by ions. The jumps of the adiabatic invariant in magnetic configurations of a stellarator are calculated. The analysis of the results obtained confirms the importance of the stochastic diffusion and demonstrates that the diffusion coefficient can considerably exceed the available result.
\end{abstract}

Ke ywords: stochastic diffusion, stellarators, particle orbits, adiabatic invariants.

\section{Introduction}

In optimized stellarators of the Wendelstein type, the prompt losses of energetic ions from superbanana orbits are prevented by modifying the magnetic configuration in order to achieve a high value of the ratio $\beta$ between the plasma and magnetic field pressures. The modification is carried out in such way that the contours of the longitudinal adiabatic invariant of locally trapped particles, $J=\oint v_{\|} d l$, should be closed inside plasma $[1,2]$. Nevertheless, the magnetic drift transforms some of those particles into locally passing ones and vice versa, i.e. a class of transitioning particles is formed. The orbits are transformed, when the particles cross the separatrix between the locally trapped and locally passing states. The separatrix crossing is accompanied by a random jump of $J$ and a transition of a particle into another bounce-averaged orbit. As a result, there arises a collisionless ${ }^{1}$ stochastic diffusion.

The coefficient of stochastic diffusion for $\alpha$-particles participating in thermonuclear reactions in a Helias reactor, i.e. the Wendelstein-type stellarator, was cal-

(c) A.V. TYKHYY, 2018

ISSN 2071-0194. Ukr. J. Phys. 2018. Vol. 63, No. 6 culated in work [3]. According to the conclusion of the cited work, the collisionless stochastic diffusion is an important mechanism giving rise to the loss of energetic ions in optimized Wendelstein-type stellarators. In particular, in the reactor concerned, this phenomenon leads to a significant fraction of $\alpha$-particles that are lost to the wall before they transfer their energy to the bulk plasma by means of Coulomb collisions. This conclusion agrees with the results of numerical simulation of particle orbits. However, in the theoretical part of work [3], the cited authors used an expression for the nonadiabatic jump of $J$ obtained in the case of a particle in a harmonic electrostatic potential that changes slowly with time [4-6]. The applicability of this expression to the behavior of transitioning particles in magnetic configurations of a stellarator has not been proved and discussed, which became a main stimulus to consider the theory of stochastic diffusion in optimized stellarators in this work. Unlike what was done in work [3], the expression for $J$-jumps in the magnetic field of a stellarator is derived here, by using the method of work [6]

1 This is a diffusion that takes place irrespective of the presence of Coulomb collisions between the particles. 
with regard for the asymmetry between locally passing particles with opposite signs of the velocity $v_{\|}$, which is caused by the presence of a magnetic field.

The structure of the work is as follows. In Section 2, a reduced Lagrangian for the motion of a guiding center in the phase space is introduced. In Section 3, this Lagrangian is applied to the magnetic configurations in stellarators of the Wendelstein type. Transitions between locally trapped and locally passing states are considered, and an expression for the probability of such transitions is derived in Section 4. In Section 5, expressions obtained in Appendix for the nonadiabatic change of $J$ at the separatrix crossing are used to derive an expression for the stochastic diffusion coefficient, which is compared with the expression presented in work [3]. The results obtained are briefly discussed and summarized in Section 6 .

\section{Lagrangian of a Guiding Center}

In the first-order approximation in the gyroradius, the motion of the guiding center of a particle in a magnetic field can be described by the following Lagrangian in the phase space [8]:

$L_{g c}=\left(\frac{e}{c} \mathbf{A}-\frac{m v_{\|}}{B} \mathbf{B}\right) \cdot \dot{\mathbf{X}}+\mu \dot{\Theta}-\mathcal{E}$,

where $\mathbf{X}$ are the guiding center coordinates; $\mu, \mathcal{E}$, $m$, and $e$ are the magnetic moment, energy, mass, and charge, respectively, of the particle; $\Theta$ is the gyrophase; $\mathbf{A}$ the vector potential; $\mathbf{B}$ the induction vector; $B=|\mathbf{B}|$ the magnetic field induction; and $v_{\|}$is the longitudinal velocity of the guiding center, which is determined by the equation $\mathcal{E}=\mu B+m v_{\|}^{2} / 2$.

Expression (1) is simple, but it is written in terms of non-canonical coordinates, thus being inconvenient for the application of standard methods of Hamiltonian mechanics. Therefore, in this and next sections, we will follow Littlejohn [9] and apply a formalism which is convenient for dealing with non-canonical coordinates. This is the so-called formalism of a $(2 n+1)$ dimensional extended phase space (see, e.g., work [10, chap. 9]). In this formalism, a mechanical system is described by a differential 1-form $\gamma$, which operates in an extended phase space. This form is called the fundamental 1-form or the Poincaré-Cartan integral invariant. The particle trajectories can be found by varying the integral $\int \gamma$, or, which is the same, by calculating the vortex lines of $\gamma$, which are the integral curves of the zero vector field $Z$ of the external derivative $d \gamma$ that satisfies the equality $d \gamma(Z)=0$. In the canonical $(\mathbf{p}, \mathbf{q}, t)$-coordinates, $\gamma$ acquires the standard form $\gamma=\mathbf{p} \cdot d \mathbf{q}-H(\mathbf{p}, \mathbf{q}, t) d t$, where $H$ is the Hamiltonian of the system, and the zero vector field $Z$ satisfies the Hamilton equations. One of the advantages of this approach consists in that the coordinates in the phase space can be non-canonical, and the time, generally speaking, is not a distinguished variable.

In our case, the extended phase space includes the coordinates $(\mathbf{X}, \mu, \Theta, \mathcal{E}, t)$, and the fundamental 1form

$\gamma_{g c}=L_{g c} d t=\left(\frac{e}{c} \mathbf{A}-\frac{m v_{\|}}{B} \mathbf{B}\right) \cdot d \mathbf{X}+\mu d \Theta-\mathcal{E} d t$

does not depend on $\Theta$ and $t$. Therefore, the magnetic moment and energy of the particle are integrals of motion, and $\Theta$ is a cyclic variable. Since we are not interested in the particle gyrophase, we can separate the conjugate pair $(\mu, \Theta)$ and, instead of $\gamma_{g c}$, consider the 1 -form

$\gamma_{g c}^{\prime}=\left(\frac{e}{c} \mathbf{A}-\frac{m v_{\|}}{B} \mathbf{B}\right) \cdot d \mathbf{X}-\mathcal{E} d t$,

which operates on the reduced phase space $(\mathbf{X}, \mathcal{E}, t)$, regarding $\mu$ to be a constant. (More formally, it is easy to show [10, Chap. 9, Section 45B] that the projections of the lines of a vortex $\gamma_{g c}$ at a given constant $\mu$ onto the hypersurface $\Theta=$ const are the vortex lines of $\gamma_{g c \cdot}^{\prime}$ )

Now, in order to calculate the jumps of adiabatic invariants, when crossing the separatrix, as well as the corresponding shifts in the radial direction, we have to know the shape of particle trajectories in the space. Therefore, we reduce the phase space once more by considering the time $t$ as a cyclic variable and separating the "conjugate pair" $(\mathcal{E}, t)$ in the same way as the pair $(\mu, \Theta)$. The particle trajectories can be obtained in the parametric form from the 1-form

$\gamma_{g c}^{\prime \prime}=\left(\frac{e}{c} \mathbf{A}-\frac{m v_{\|}}{B} \mathbf{B}\right) \cdot d \mathbf{X}$,

which operates in a three-dimensional reduced phase space consisting of only $\mathbf{X}$, with $\mathcal{E}$ and $\mu$ being now regarded as constants.

\section{Magnetic Configuration}

The Boozer coordinates $(\psi, \vartheta, \varphi)$, where $\psi$ is a toroidal magnetic flux, and $\vartheta$ and $\varphi$ are the poloidal 
and toroidal, respectively, angular variables, will be used below. In these coordinates, we have the relationship $\vartheta-\iota(\psi) \varphi=$ const at the magnetic force line, where $\iota=d \psi_{p} / d \psi$ is the rotation transform and $\psi_{p}$ the poloidal magnetic flux. Furthermore, in the same coordinates, $\mathbf{A}=\psi \nabla \vartheta-\psi_{p} \nabla \varphi$ and $B_{\varphi} \approx$ $\approx B R \gg\left(B_{\psi}, B_{\vartheta}\right)$. We will consider a magnetic configuration that is typical of Wendelstein-type stellarators, where the mirror and helical harmonics dominate in $B$, and the number of stellarator periods $N$ is large $(\iota / N \ll 1)$. For $B$, we take the expression

$\frac{B}{\bar{B}}=1+\epsilon_{0}-\epsilon_{t} \cos \vartheta+\epsilon_{m} \cos N \varphi-\epsilon_{h} \cos (N \varphi-\vartheta)$,

where $\bar{B}$ is the average magnetic field induction at the stellarator axis; $\epsilon_{h}, \epsilon_{m}$, and $\epsilon_{t}$ are the amplitude of the helical, mirror, and toroidal, respectively, harmonics; and $\epsilon_{0}$ describes the modification of the vacuum magnetic configuration due to the finite plasma pressure. Those amplitudes, as well as $\epsilon_{0}$, are $o(1)$ functions of $\psi$. Then, Eq. (4) can be reduced to

$\gamma_{g c}^{\prime \prime}=\frac{e}{c}\left(\psi d \vartheta-\psi_{p} d \varphi\right)-m v_{\|} R d \varphi=\frac{e}{c} \psi d \vartheta-p_{\varphi} d \varphi$,

where $p_{\varphi}=(e / c) \psi_{p}+m v_{\|} R$. Formally, 1-form (6) describes a one-dimensional Hamiltonian system with a time-dependent Hamiltonian, in which the variables $\vartheta, \varphi$, and $\psi$ play the role of time, coordinate, and energy, respectively; and $p_{\varphi}$ is a canonical momentum conjugate to $\varphi$. Since $N \gg 1$, the dependence of $\varphi$ on $\vartheta$ can be considered as a perturbation. From the physical viewpoint, we consider the motion of particles in local magnetic wells associated with the mirror and helical $B$-harmonics. The period of those harmonics is of an order of $2 \pi / N$, whereas the period of the toroidal harmonic equals $2 \pi / \iota$. For convenience, we combine the mirror and helical $B$-harmonics:

$\frac{B}{\bar{B}}=1+\epsilon_{0}-\epsilon_{t} \cos \vartheta+\epsilon_{h m} \cos N(\varphi-\chi)$,

where $\epsilon_{h m}$ and $\chi$ are functions of $\psi$ and $\vartheta$. Then, for $p_{\varphi}$, we obtain

$p_{\varphi}=-\frac{e}{c} \psi_{p} \mp m \omega_{B} \bar{\rho}_{l} R \sqrt{\epsilon_{h m}\left(\kappa^{2}-\cos ^{2} N(\varphi-\chi) / 2\right)}$,

where $\omega_{B}=e \bar{B} /(m c), \bar{\rho}_{l}$ is the Larmor radius of the particle at the magnetic axis, $R$ the major plasma radius,

$\kappa^{2}=\frac{\alpha-\left(\epsilon_{0}-\epsilon_{t} \cos \vartheta-\epsilon_{h m}\right)}{2 \epsilon_{h m}}$,

ISSN 2071-0194. Ukr. J. Phys. 2018. Vol. 63, No. 6

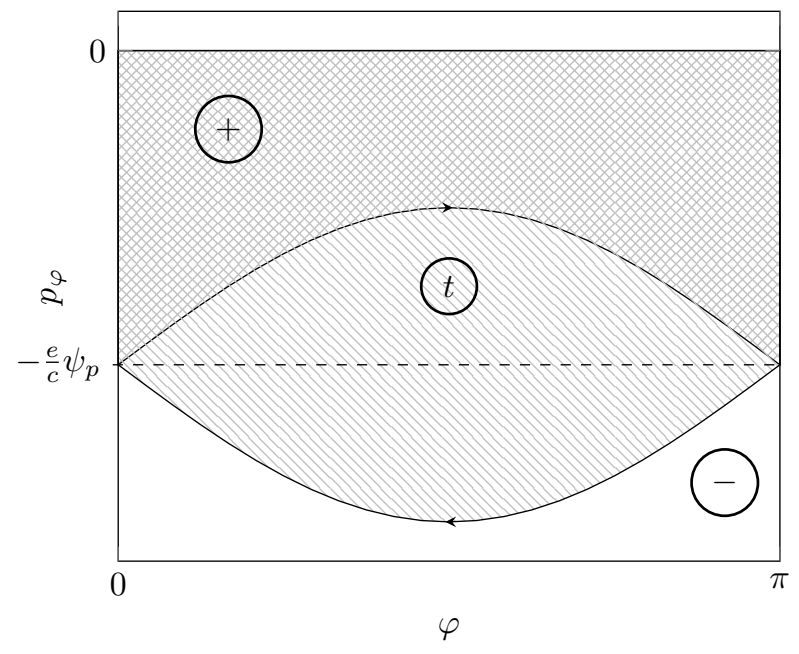

Fig. 1. Phase portrait of a "shifted" nonlinear pendulum [see Eq. (6)]. The $(+)$ and $(-)$ locally passing states occupy the phase space regions located above the upper separatrix branch and under the lower one, respectively. The phase space regions that are included into $S_{+}$and $S_{-}$are indicated by slanted hatching. The $S_{+}$and $S_{-}$regions have opposite signs, because the contours at their boundaries are traced in opposite directions

and $\alpha=\mathcal{E} /(\mu \bar{B})-1$ is the pitch angle parameter. After the above simplifications, the system described by Eq. (6) turns out similar to a nonlinear pendulum. In particular, the trajectories of locally trapped particles correspond to the pendulum libration, and the trajectory of locally passing particles to the pendulum rotation.

The phase portrait of this system (see Fig. 1) is the phase portrait of a nonlinear pendulum shifted by $\frac{e}{c} \psi_{p} / N$. The action variable of this system is the standard adiabatic invariant $J_{\|}$. For locally trapped particles, $\kappa^{2}<1$, and we have one branch

$I_{t}=\frac{1}{2 \pi} \oint p_{\varphi} d \varphi=I_{s} f(\kappa)$

where

$I_{s}=\frac{4}{\pi N} m \omega_{B} \bar{\rho}_{l} R \sqrt{\epsilon_{h m}}$,

$f(\kappa)=E(\kappa)-\left(1-\kappa^{2}\right) K(\kappa)$,

and $K$ and $E$ are the complete elliptic integrals of the first and second kinds, respectively. For locally passing particles, integration is carried out over the whole period $2 \pi / N$, and the adiabatic invariant 


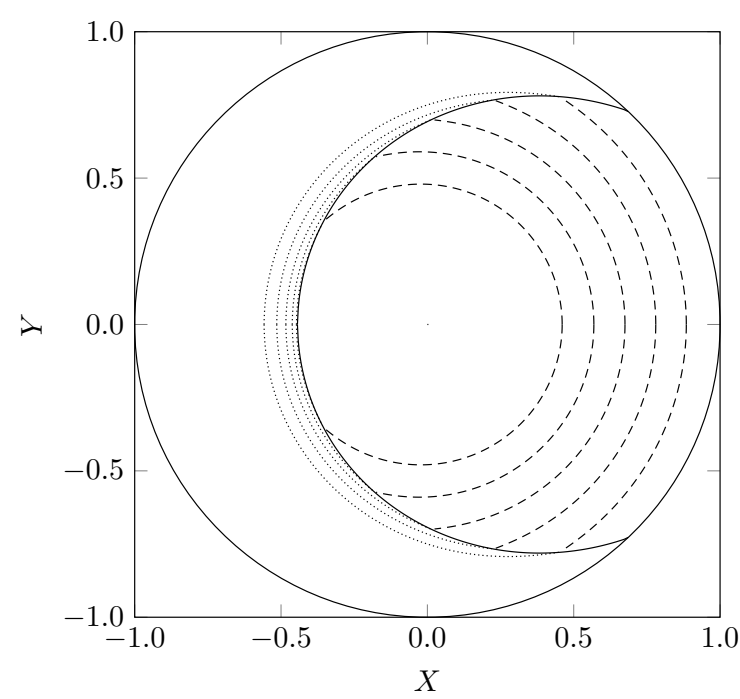

Fig. 2. $J_{\|}=$const contours for locally trapped (dotted curves) and locally passing (dashed curves) particles with $\alpha=0.2$. The solid curve corresponds to the separatrix $\kappa^{2}=1$

has two branches

$I_{ \pm}=\mp \frac{e}{c} \frac{\psi_{p}}{N}+\frac{1}{2} I_{s} f(\kappa)$,

where $f(\kappa)$ is the same function as in Eq. (10), but continued into the region $\kappa^{2}>1$, where, using a standard transformation, it can be rewritten in the form $f(\kappa)=\kappa E\left(\kappa^{-1}\right)$. Expressions (10) and (11) coincide with the results of work [3]. The bounce-averaged trajectories of particles correspond to $I_{i}=$ const contours in the $(\psi, \vartheta)$-plane (Fig. 2). The subscript $i$ equals "+" or "-", corresponding to the sign of $v_{\|}$ for locally passing particles and to the sign of $t$ for locally trapped particles.

The contour $\kappa^{2}=1$ in the $(\psi, \vartheta)$-plane is a separatrix between locally passing and locally trapped particles with a given pitch angle. The stochastic diffusion is a result of the accumulation of $I_{i}$-jumps taking place when the bounce-averaged trajectory of a particle crosses the separatrix, where the adiabaticity of $I_{i}$ breaks down.

\section{Transitions}

For the magnetic configurations of Wendelstein-7X, there are two transition points in the whole interval of the values for the pitch-angle parameter $\alpha$, for which transitional orbits are available; the transition points are located symmetrically with respect to the plane $\vartheta=0^{2}$. The types and probabilities of the transitions taking place in the system are determined by the relative changes of the phase volume occupied by each state.

The phase space occupied by locally trapped particles is an area circumscribed by the separatrix:

$\frac{S_{t}(\vartheta)}{2 \pi}=\left.I_{t}\right|_{\kappa^{2}=1}=I_{s}$.

Hereafter, except if otherwise indicated, all the quantities and derivatives are calculated at $\kappa^{2}=1$. The phase volumes occupied by locally passing states are unlimited, because the quantity $\left|v_{\|}\right|$can acquire arbitrarily large values. However, we are not interested in the absolute values of those phase volumes, but in their changes induced by a shift of the separatrix with the "time" $\vartheta$. Following work [6], we use the notation $S_{ \pm}$for the areas between the corresponding separatrix branch and the axis $\varphi$, so that $S_{t}=S_{+}+S$. Then

$\frac{S_{ \pm}(\vartheta)}{2 \pi}=\left.I_{ \pm}\right|_{\kappa^{2}=1}=\mp \frac{e}{c} \frac{\psi_{p}}{N}+\frac{1}{2} I_{s}$

The phase volume changes are given by the expression

$\Theta_{i}=\frac{d S_{i}}{d \vartheta}=\frac{\partial S_{i}}{\partial \vartheta}-\frac{\partial S_{i}}{\partial \psi} \frac{\partial \kappa^{2}}{\partial \vartheta}\left(\frac{\partial \kappa^{2}}{\partial \psi}\right)^{-1}=$

$=\left(\frac{\partial \kappa^{2}}{\partial \psi}\right)^{-1}\left\{\kappa^{2}, S_{i}\right\}$,

where the notation

$\{F, G\} \equiv \frac{\partial F}{\partial \psi} \frac{\partial G}{\partial \vartheta}-\frac{\partial F}{\partial \vartheta} \frac{\partial G}{\partial \psi}$

is used. Note that the phase volume occupied by the corresponding state decreases when $\Theta_{ \pm}>0$. For locally trapped particles,

$\frac{\Theta_{t}}{2 \pi}=\left(\frac{\partial \kappa^{2}}{\partial \psi}\right)^{-1}\left\{\kappa^{2}, I_{s}\right\}=$

$=\left(\frac{\partial \kappa^{2}}{\partial \psi}\right)^{-1} \frac{I_{s}}{4 \epsilon_{h m}^{2}}\left\{\kappa^{2}, \epsilon_{h m}^{2}\right\}=\frac{I_{s}}{2 \epsilon_{h m}^{2}} \frac{\delta}{\delta_{1}} \sin \vartheta$,

where $r$ is the dimensionless pseudoradial coordinate determined from the equation $\psi=\bar{B} a^{2} r^{2} / 2, a$ is the minor plasma radius, and

$\delta=\epsilon_{h} \epsilon_{m}\left(\epsilon_{0}^{\prime}-\epsilon_{t}^{\prime} \cos \vartheta\right)-\epsilon_{t}\left(\epsilon_{h m}^{2}\right)^{\prime} / 2$,

2 This statement is incorrect in the case where $\epsilon_{m}^{\prime}>\epsilon_{h}^{\prime}$ and, simultaneously, $\epsilon_{0}^{\prime}<\epsilon_{t}$. But this situation is not realized for any of the configurations given in work [11].

ISSN 2071-0194. Ukr. J. Phys. 2018. Vol. 63, No. 6 
$\delta_{1}=\epsilon_{0}^{\prime}+\epsilon_{h m}^{\prime}-\epsilon_{t}^{\prime} \cos \vartheta$

where the primes mean the derivatives with respect to $r$. For locally passing particles,

$$
\begin{aligned}
& \frac{\Theta_{ \pm}}{2 \pi}=\left(\frac{\partial \kappa^{2}}{\partial \psi}\right)^{-1}\left(\left\{\kappa^{2}, \mp \frac{e}{c} \frac{\psi_{p}}{N}\right\}+\frac{1}{2}\left\{\kappa^{2}, I_{s}\right\}\right)= \\
& =\frac{1}{2} \frac{\Theta_{t}}{2 \pi} \pm\left.\frac{e}{c} \frac{\iota}{N} \frac{d \psi}{d \vartheta}\right|_{\kappa^{2}=1} .
\end{aligned}
$$

For convenience, let us introduce the pseudoprobability of the transition

$$
\tilde{P}=\frac{2 \Theta_{t}}{\Theta_{+}-\Theta_{-}}=\frac{2}{\pi \iota r} \frac{\bar{\rho}_{l} R}{a^{2}} \frac{\delta}{\sqrt{\epsilon_{h m}}\left(\epsilon_{h} \epsilon_{m}+\epsilon_{t} \epsilon_{h m}\right)} .
$$

Our numerical estimates show that, for the thermonuclear $\alpha$-particles in a Helias reactor, the parameter $\tilde{P}$ is positive and less than 0.1 over the whole plasma volume. This quantity is even less for the energetic ions of a neutral injection in Wendelstein-7X. Accordingly, the changes in the phase volume of locally passing particles always have opposite signs, being close by the absolute value:

$$
\frac{\Theta_{+}}{\Theta_{-}}=\frac{\left(\Theta_{+}-\Theta_{-}\right) / 2+\Theta_{t} / 2}{\left(\Theta_{-}-\Theta_{+}\right) / 2+\Theta_{t} / 2}=-\frac{1-\tilde{P}}{1+\tilde{P}}=O(1),
$$

whereas the phase flux of locally trapped particles is $O(\tilde{P})$.

The signs in Eq. (11) are so determined that $\Theta_{+}>$ $>0$ at the upper transition point $(0<\vartheta<\pi)$. At this point, the phase volume occupied by $(+)$ locally passing particles decreases, and the volume occupied by $(-)$ locally trapped particles increases. Therefore, the $(+)$ locally passing particles transform into the locally trapped ones with the probability $P=\Theta_{t} / \Theta_{+}=\tilde{P} /(1-\tilde{P} / 2) \approx \tilde{P}$. This result coincides with formula (25) in work [3], although it was obtained in a different way. The other $(+)$ locally passing particles change the drift direction and transform into $(-)$ locally passing ones. At the lower transition point, the signs of $\Theta_{i}$ 's change to the opposite ones. Then the phase volume occupied by locally trapped particles decreases, and, accordingly, they "escape" from the local magnetic wells with a probability of 1 and become locally passing ones. At the same time, the $(-)$ locally passing particles become $(+)$ locally passing ones and drift backward to the upper transition point.

\section{Diffusion Coefficient}

In order to derive expressions for the adiabaticinvariant jumps near the transition points, the theory of separatrix crossing developed by Neishtadt [6] will be used. Since the phase portrait of a non-linear pendulum differs from that of the system considered in work [6], the expressions obtained there cannot be applied directly. A somewhat cumbersome calculation of the jump carried out in the framework of [6] (see Appendix) brings us to the following expression for the jump of the adiabatic invariant $I_{t}$ at the transition between the locally trapped and locally passing states:

$$
\begin{aligned}
& 2 \pi \delta J_{+\rightarrow t}=\frac{\Theta_{t}}{\Theta_{+}}\left(b_{+}^{\prime} \Theta_{t}-b_{t}^{\prime} \Theta_{+}\right) \xi+ \\
& +a \Theta_{t} \ln \frac{\Gamma(\xi) \Gamma\left(\xi+\frac{\Theta_{+}}{\Theta_{t}}\right) \Gamma\left(\frac{\Theta_{t}}{\Theta_{+}}(1-\xi)\right)}{(2 \pi)^{\frac{3}{2}}\left|\xi-1+\frac{\Theta_{+}}{\Theta_{t}}\right|},
\end{aligned}
$$

where $a$ and $b_{i}^{\prime}$ are coefficients determined from the expansion of $I_{i}$ near the separatrix, and $0<\xi<1$ is a transition parameter that corresponds to the exact particle coordinate at the moment, when the separatrix is crossed. The terms independent of $\xi$ are omitted, because they do not contribute to the diffusion coefficient. For the next separatrix crossings, the parameter $\xi$ can be regarded as a uniformly distributed random variable, so that the adiabatic invariant will undergo a stochastic fluctuation with the mean square step $\left\langle\left(2 \pi\left(\delta J_{+\rightarrow t}-\left\langle\delta J_{+\rightarrow t}\right\rangle\right)\right)^{2}\right\rangle$, where the angle brackets denote the averaging over $\xi$.

Note that, in a special case where

$\Theta_{+}=\Theta_{-}=\Theta_{t} / 2, \quad b_{+}=b_{-}$,

expression (13) is reduced to $a \Theta_{t} \ln \sin \xi$, and the mean-square step of the adiabatic invariant equals

$\left\langle\left(2 \pi\left(\delta J_{+\rightarrow t}-\left\langle\delta J_{+\rightarrow t}\right\rangle\right)\right)^{2}\right\rangle=\left(a \Theta_{t}\right)^{2} \pi^{2} / 12$.

This case corresponds to the problem of a particle in a harmonic electric potential that slowly changes in time [4-6]. However, conditions (14) mean that, depending on the $\Theta_{t}$-sign, the "passing" particles become trapped by the potential, and the trapped particles transform into passing ones, with a probability equal to 1 , and there are no transitions between locally passing states. In our case, on the contrary, the probability of the transformation at the upper transition point $P \ll 1$. At the same time, at the lower 
transition point, the phase flux of locally trapped particles that transform into locally passing ones comprises a small $(\sim P)$ fraction of the flux of locally passing particles that change the drift direction to the opposite one. Furthermore, conditions (14) demand that there should be a symmetry between the locally passing states, which is absent in our case due to the presence of the term $-\frac{e}{c} \psi_{p}$ in Eq. (8) ${ }^{3}$.

In the case concerned, we have $\tilde{P} \ll 1$, and the mean-square step is given by Eq. (31). Neglecting the small correlation term and making the approximation $\Theta_{t} / \Theta_{+} \approx \tilde{P}$ in the leading-order in $\tilde{P}$, we obtain

$$
\begin{aligned}
& \left\langle\left(2 \pi\left(\delta J_{+\rightarrow t}-\left\langle\delta J_{+\rightarrow t}\right\rangle\right)\right)^{2}\right\rangle \approx M_{2}\left(a \Theta_{t}\right)^{2}+ \\
& +\frac{1}{12}\left(-a \Theta_{t} \ln \tilde{P}+\tilde{P}\left(b_{+}^{\prime} \Theta_{t}-b_{t}^{\prime} \Theta_{+}\right)\right)^{2}
\end{aligned}
$$

where $M_{2} \approx 0.77$ is a constant obtained, when calculating the integrals with $\Gamma$-functions.

Now, we have to know expressions for the coefficients $a$ and $b_{i}^{\prime}$. Following the method of work [6], we expand the adiabatic invariants in a series in the "energy distance" from the separatrix, $h=\psi-\psi_{s}$, at a fixed "time" $\vartheta$. Here, $\psi_{s}$ is the separatrix position determined from the equation $\kappa^{2}\left(\psi_{s}, \vartheta\right)=1$. Near $\kappa^{2}=1$, the function $f$ can be estimated as

$f(\kappa)=1-\frac{s}{4}\left(1+\ln \frac{16}{|s|}\right)+O\left(s^{2} \ln |s|\right)$,

where the parameter

$s=1-\kappa^{2} \approx-\frac{\partial \kappa^{2}}{\partial \psi} h$

is positive for locally trapped particles and negative for locally passing ones. Then, for $I_{ \pm}$, we obtain

$$
\begin{aligned}
& I_{ \pm} \approx \mp \frac{e}{c} \frac{\psi_{p}}{N} \mp \frac{e}{c} \frac{\iota}{N} h+\frac{1}{2} I_{s}+\frac{1}{2} \frac{\partial I_{s}}{\partial \psi} h- \\
& -\frac{1}{8} I_{s} \frac{\partial \kappa^{2}}{\partial \psi} h \ln \left|\frac{1}{16 e} \frac{\partial \kappa^{2}}{\partial \psi} h\right|,
\end{aligned}
$$

3 Physically, this asymmetry means that, at the upper transition point, the trajectory section of a locally trapped particle that is close to the separatrix (-) branch is "longer" in the $\vartheta$ direction in comparison with the trajectory section close to the $(+)$ branch. At the lower transition point, the relationship is reversed. and the coefficients in Eq. (21) are given by the expressions

$\frac{a_{ \pm}}{2 \pi} \equiv \frac{a}{2 \pi}=\frac{1}{8} I_{s} \frac{\partial \kappa^{2}}{\partial \psi}$

$\frac{b_{ \pm}}{2 \pi}=\mp \frac{\iota}{N}+\frac{1}{2} \frac{\partial I_{s}}{\partial \psi}-\frac{1}{8} I_{s} \frac{\partial \kappa^{2}}{\partial \psi} \ln \left|\frac{1}{16} \frac{\partial \kappa^{2}}{\partial \psi}\right|$.

Since $I_{t}=I_{+}+I_{-}$, it follows for locally trapped particles that $b_{t}=b_{+}+b_{-}$and $a_{t}=2 a$. Introducing the notation $b_{i}^{\prime}=b_{i}-a_{i} \ln \left|\Theta_{i}\right|$, keeping only the leadingorder terms in $\tilde{P}$, and performing some transformations, we obtain

$$
\begin{aligned}
& b_{+}^{\prime} \Theta_{t}-b_{t}^{\prime} \Theta_{+}=b_{+}^{\prime} \Theta_{-}-b_{-}^{\prime} \Theta_{+}= \\
& =2 \pi \Theta_{+}\left(\frac{\iota}{N} \tilde{P}-\frac{\partial I_{s}}{\partial \psi}+\frac{I_{s}}{4} \frac{\partial \kappa^{2}}{\partial \psi} \ln \left|\frac{\pi}{8}\left\{\kappa^{2}, \frac{\psi_{p}}{N}\right\}\right|\right) .
\end{aligned}
$$

Additionally, we have to find a relation between the adiabatic invariant change and the radial shift of the point, where the drift trajectory of the particle crosses the separatrix:

$\delta \psi_{+\rightarrow t}=\left.\frac{d \psi}{d I_{i}}\right|_{\kappa^{2}=1} \delta J_{+\rightarrow t}$,

where

$\left.\frac{d \psi}{d I_{i}}\right|_{\kappa^{2}=1}=\left.\frac{d \psi}{d \vartheta}\right|_{\kappa^{2}=1}\left(\frac{d I_{i}}{d \vartheta}\right)^{-1}=\frac{\partial \kappa^{2}}{\partial \vartheta}\left(\frac{\partial \kappa^{2}}{\partial \psi}\right)^{-1} \frac{2 \pi}{\Theta_{i}}$.

By combining the formulas obtained above, we arrive at the expression for the mean-square radial shift of the particle at the transition between the locally passing and locally trapped states:

$\left\langle\left(\delta \psi_{+\rightarrow t}-\left\langle\delta \psi_{+\rightarrow t}\right\rangle\right)^{2}\right\rangle=\left(\frac{\pi I_{s}}{4} \frac{\partial \kappa^{2}}{\partial \vartheta}\right)^{2}\left(M_{2}-\frac{Q^{2}}{12}\right)$

where

$Q=\ln \tilde{P}+2 \ln \left|\frac{\pi}{8}\left\{\kappa^{2}, \frac{\psi_{p}}{N}\right\}\right|+8 \frac{1}{I_{s}} \frac{\partial I_{s}}{\partial \vartheta}\left(\frac{\partial \kappa^{2}}{\partial \vartheta}\right)^{-1}$.

The coefficient of stochastic diffusion, which arises as a result of the jumps of adiabatic invariants at those transitions, can be evaluated by the expression

$D=\left(\frac{d r}{d \psi}\right)^{2}\left\langle\left(\delta \psi_{+\rightarrow t}-\left\langle\delta \psi_{+\rightarrow t}\right\rangle\right)^{2}\right\rangle \frac{2 P}{\tau}$

ISSN 2071-0194. Ukr. J. Phys. 2018. Vol. 63, No. 6 
where $\tau$ is the average time between the particle transformations at the upper transition point. The multiplier $2 P$ takes into account that, every time when the particle transforms into a locally trapped one with the probability $P$, two transitions take place between locally passing and locally trapped states. Using an estimate for $\tau$ from work [3], we obtain

$D=D^{(0)}\left(\frac{\pi^{2}}{12}\right)^{-1}\left(M_{2}-\frac{Q^{2}}{12}\right)$

where

$D^{(0)}=\frac{\pi^{3} R^{2} \omega_{B} \bar{\rho}_{l}^{4}}{3 N^{2} a^{4} r^{3}} \epsilon_{h m}\left(\frac{\partial \kappa^{2}}{\partial \vartheta}\right)^{2} \frac{\delta}{\epsilon_{h} \epsilon_{m}+\epsilon_{h m} \epsilon_{t}+\delta / \delta_{1}}$

is the coefficient of stochastic diffusion, which is given in work $[3$, formula $(36)]$. The corresponding expression can be obtained by substituting Eq. (15) rather than Eq. (16) into Eq. (17).

Expression (20) obtained for the stochastic diffusion coefficient makes allowance only for the contributions of transitions between locally trapped and locally passing states. It neglects a possible contribution to the stochastic diffusion from the transformation of particles between two locally passing states, i.e. from the change of the drift direction of locally passing particles to the opposite one. The latter contribution is given by the expression

$D_{ \pm}=\left(\frac{d r}{d \psi}\right)^{2}\left\langle\left(\delta \psi_{+\rightarrow-}-\left\langle\delta \psi_{+\rightarrow-}\right\rangle\right)^{2}\right\rangle \frac{2}{\tau}$

where the mean-square step of the adiabatic invariant is given by Eq. (28). This contribution is not taken into consideration, because it is unclear whether the transition parameter can be regarded as a uniformly distributed random variable at every next transition. Furthermore, $D_{ \pm}$has the same order of magnitude as $D^{(0)}$ has; therefore, it can hardly affect any conclusions concerning the influence of the stochastic diffusion in our case.

In order to compare $D$ and $D^{(0)}$, let us calculate the numerical values of both coefficients for the same magnetic configuration and particle parameters as in work [3]. Namely, these are thermonuclear $\alpha$-particles in a Helias reactor with $\langle\beta\rangle=4.7 \%$, $B=4.75 T$, and $a / \bar{\rho}_{l}=30$. The results of calculations are depicted in Figs. 3 and 4 . The general behavior of the $D$-dependences on $r$ and $\alpha$ remains the same, but the values calculated in accordance with

ISSN 2071-0194. Ukr. J. Phys. 2018. Vol. 63, No. 6

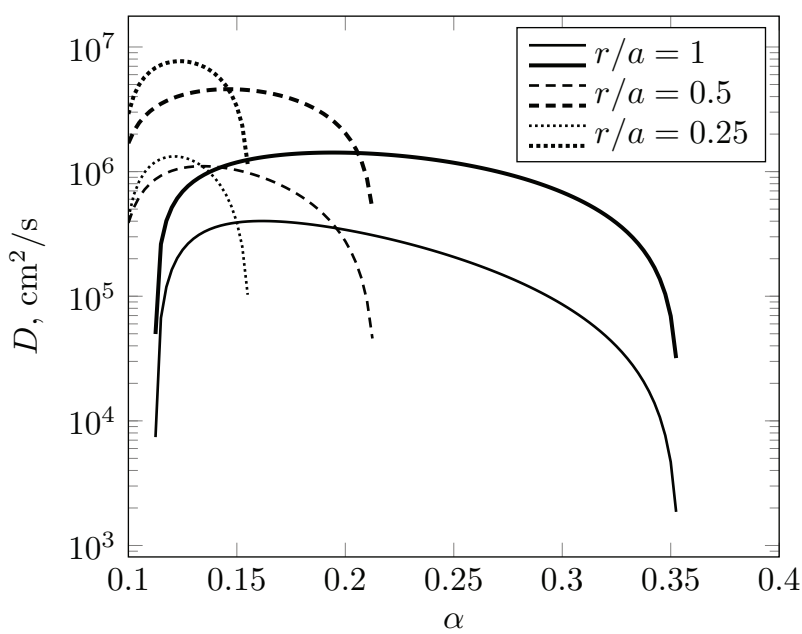

Fig. 3. Dependences of the diffusion coefficients $D$ (bold curves) and $D^{(0)}$ (thin curves) on the pitch-angle parameter $\alpha=\mathcal{E} / \mu \bar{B}-1$ for various $r / a$-ratios

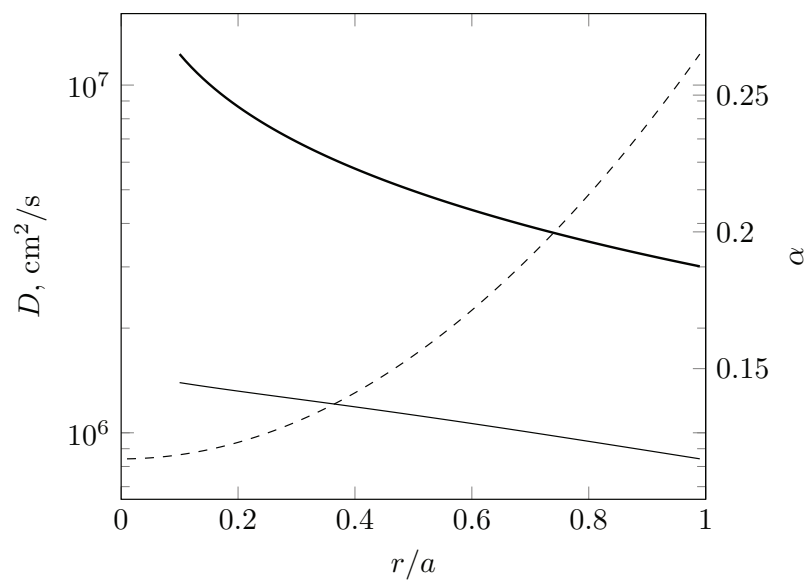

Fig. 4. Dependences of the diffusion coefficients $D$ (bold curve) and $D^{(0)}$ (thin curve) on the radius at $\alpha=\left(\alpha_{\min }+\right.$ $\left.+\alpha_{\max }\right) / 2$. The dashed curve is the dependence of the average $\alpha$ on the radius

formula (20) exceed the previous estimates by several times, especially near $\alpha_{\max }$, i.e. for weakly transitioning particles.

The expression for $D^{(0)}$ gives qualitatively correct results, because the jumps of the adiabatic invariant are the product of the phase flux of particles across the separatrix and a dimensionless multiplier that involves both the intervals, in which the transition parameters are distributed, and the phase space structure near the separatrix (in particular, the number and the order of the particle trajectory ap- 
proaches to the hyperbolic points). The phase fluxes between the states depend on neither the transition parameters for separate particles nor the phase space structure near the separatrix, being determined directly from the expressions for adiabatic invariants (12). The probabilities of transitions also depend only on those fluxes. Therefore, our expression obtained for the probability of the transition coincides with that calculated in work [3].

\section{Conclusions}

To summarize, an expression has been derived for the coefficient of stochastic diffusion of transitioning particles in stellarators of the Wendelstein type. The formula is based on the expression for the jumps of the adiabatic invariant in the magnetic field of a stellarator and considers the asymmetry between locally passing particles with opposite signs of $v_{\|}$created by the magnetic field. It is shown that the conclusions of work [3] remain qualitatively correct, despite that the expression was used for the particle adiabatic invariant jumps in a harmonic electric potential slowly varying in time [4-6]. Nevertheless, the diffusion coefficient calculated according to the new formula turns out several times higher than the previous estimates. The discrepancy is especially noticeable for weakly transitioning particles.

The author is grateful to Ya.I.Kolesnichenko and V.S.Marchenko for valuable discussions and comments.

\section{APPENDIX. Separatrix crossing}

\section{in the case of "shifted" nonlinear pendulum}

In this Appendix, using the method described in work [6], expressions are derived for the change of adiabatic invariants at a slow crossing of the separatrix in a Hamiltonian system, whose phase portrait is depicted in Fig. 1 and whose Hamiltonian $H(p, q, \varepsilon t)$ slowly depends on the time. For convenience, the Hamiltonian $H(p, q, \varepsilon t)$ is assumed to be zero at the separatrix and negative in the "trapped" region, as it takes place for an ordinary nonlinear pendulum. Similarly to the main text, the phase volumes of the states are denoted as $S_{i}$, and the corresponding phase fluxes as $\Theta_{i}=\dot{S}_{i}$.

We assume that $\Theta_{+} \Theta_{-}<0$ and, without loss of generality, put $\Theta_{+}>0$. Then the following transitions among three states take place:

$\Theta_{t}<0:\left\{\begin{array}{lll}(+) \rightarrow & (-) & (a), \\ (t) \rightarrow & (-) & (b),\end{array}\right.$

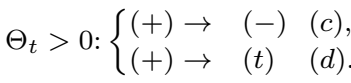

The difference between transitions (a) and (c) consists in that all $(+)$ passing trajectories transform into $(-)$ passing ones in case $(\mathrm{a})$, and some $(+)$ passing trajectories become trapped ones in case (c).

Let us determine the coefficients $a_{i}, b_{i}$, and $S_{i}$ in the expansion of the adiabatic invariants $I_{i}$ near the separatrix:

$2 \pi I_{i}=\oint p d q=S_{i}-a_{i} h \ln |h|+\left(b_{i}+a_{i}\right) h+O\left(h^{2} \ln |h|\right)$,

where $h$ is the energy of the system at the trajectory. Accordingly,

$\oint_{H=h} \frac{\partial H}{\partial t} d t=-2 \pi \frac{\partial I_{i}}{\partial t}=-\Theta_{i}+O(h \ln |h|)$.

The period of the particle motion along a trajectory near the separatrix is given by the expression

$T_{i}=2 \pi\left(\frac{\partial h}{\partial I_{i}}\right)^{-1}=b_{i}-a_{i} \ln |h|+O(h \ln |h|)$.

If $\dot{H} \neq 0$, the adiabatic invariants are not constant, and their deviations from the average value over the period are of the $O(\varepsilon)$-th order. Neishtadt [6] uses smoothed adiabatic invariants $J_{i}=I_{i}+u_{i}$, where

$u_{i}=\frac{1}{2 \pi} \int_{0}^{T_{i}}\left(\frac{T_{i}}{2}-t\right) \frac{\partial H}{\partial t} d t$,

which deviate from the average value over the period by an order of $O\left(\varepsilon^{2}\right)$. The behavior of the corrections $u_{i}$ near the separatrix depends on how many times the trajectory approaches the hyperbolic points during the period. In our case, as well as in the case considered in work [6], the "passing" trajectories approach a single hyperbolic point one time per period, and $2 \pi u_{ \pm}=d_{ \pm}+O(\sqrt{h} \ln |h|)$, where $d_{ \pm}$are constants. At the same time, for "trapped" trajectories, which approach the single hyperbolic point twice per period,

$2 \pi u_{t}=\left(T_{+} \Theta_{-}-T_{-} \Theta_{+}\right) / 2+d_{t}+O(\sqrt{h} \ln |h|)$,

where $d_{t}=d_{+}+d_{-}$. Below, the evaluations of rejected terms, which are analogous to those made in work [6], are omitted.

At first, let us consider transition (a). As long as the system is far from the separatrix, $I_{+}=I_{+\infty}$ and $J_{+}=J_{+\infty}$ remain almost unchanged. We can determine the time of the pseudocrossing $\tau_{*}$ [7] as a root of the equation $S_{+}\left(\tau_{*}\right)=2 \pi J_{+\infty}$. This is the time that would be required for the trajectory to cross the separatrix, if the adiabatic invariant remained unchanged until the very intersection. We can also determine the pseudofinal value of $J_{-}$after the transition: $2 \pi J_{-*}=S_{-}\left(\tau_{*}\right)$.

From Eq. (22), one can see that, after every period, the system energy decreases by $\Theta_{+}$, i.e. $h_{n}=h_{0}+n \Theta_{+}$, where the sequential points of the maximum trajectory approach to the hyperbolic point are enumerated from the last approach before the crossing. The corresponding time moments of the maximum approaches are connected by the equality

$\tau_{+\mid n-1}=\tau_{+\mid n}+\frac{1}{2}\left(T_{+}\left(h_{n}\right)+T_{+}\left(h_{n-1}\right)\right)$,

ISSN 2071-0194. Ukr. J. Phys. 2018. Vol. 63, No. 6 
because every interval between the sequential approaches consists of a slow movement away from the hyperbolic point, when the system energy is close to $h_{n}$, and a slow movement toward it, when the system energy is close to $h_{n-1}$. The time required for the trajectory to pass past the hyperbolic point at an almost constant energy value $h$ dominates in the motion period $T_{i}(h)$. According to the definition of the maximum approach before the separatrix crossing, this energy $0<h_{0}<\Theta_{+}$.

Let us define the dimensionless transition parameter $\xi_{+}=$ $=h_{0} / \Theta_{+}$. When the trajectory crosses the separatrix and enters the $(-)$ region, it is close at first to the separatrix branch that separates the $(+)$ and $(t)$ regions. Afterward, it approaches the hyperbolic point and moves away from it along the separatrix branch that separates the $(t)$ and $(-)$ regions. Accordingly, the system energy near the hyperbolic point is equal to $g_{*}=h_{0}-\Theta_{+}$at the moment, when the trajectory approaches it from the $(t)$ region, and to $g_{0}=g_{*}-\Theta_{-}=h_{0}-\Theta_{t}$, if it approaches the hyperbolic point from the $(-)$ region. The value of $g_{0}$ is positive (as it should be in the (-) "passing" state), because $\Theta_{t}<0$. The transition parameter in the $(-)$ region, $\xi_{-}=-g_{0} / \Theta_{-}$, is related to $\xi_{+}$by the obvious relation $\Theta_{+}\left(1-\xi_{+}\right)=-\Theta_{-}\left(1-\xi_{-}\right)$.

Note that, although $0<\xi_{+}<1$, an analogous inequality for $\xi_{-}$is, generally speaking, is invalid: some fraction of the phase flux into the $(-)$ region is composed of trajectories that transit into this region from a "trapped" state. By adding the contributions to the time that is required for the trajectory to get from $h_{0}$ to $g_{0}$ and recalling that, in the $(t)$ state, the trajectory approaches the hyperbolic point twice per period, we obtain

$\tau_{-\mid 0}=\tau_{+\mid 0}+\frac{1}{2} T_{+}\left(h_{0}\right)+\frac{1}{2} T_{t}\left(g_{*}\right)+\frac{1}{2} T_{-}\left(g_{0}\right)$.

Between the next moments of the maximum approach to the hyperbolic point by the trajectory that now moves away from the separatrix, the system energy increases by $\Theta_{-}$per period, i.e. $g_{n}=g_{0}-n \Theta_{-}$. Similar considerations show that the times of the sequential moments of the maximum approach to the hyperbolic point in the $(-)$ region are connected by the relation $\tau_{-\mid n}=\tau_{-\mid n-1}+\frac{1}{2}\left(T_{-}\left(g_{n-1}\right)+T_{-}\left(g_{n}\right)\right)$.

By substituting the approximate expressions for $T_{i}$ and $I_{i}$ near the separatrix and transfroming the recurrent relations into sums, it is easy to obtain

$\tau_{+\mid 0} \approx \tau_{+\mid n}+n\left(b_{+}-a_{+} \ln \Theta_{+}\right)-$

$-\frac{1}{2} a_{+} \ln \frac{\xi_{+}+n}{\xi_{+}}-a_{+} \ln \frac{\Gamma\left(\xi_{+}+n\right)}{\Gamma\left(\xi_{+}\right)}$

and, according to Eq. (21),

$2 \pi\left(J_{+0}-J_{+n}\right) \approx \Theta_{+}\left(\tau_{+\mid 0}-\tau_{+\mid n}\right)-\left(a_{+}+b_{+}\right) n \Theta_{+}-$

$-a_{+}\left(h_{0} \ln h_{0}-h_{n} \ln h_{n}\right)$.

Let us consider a moment, when the trajectory is rather far from the separatrix, i.e. $n \gg 1$. Applying the Stirling formula $\Gamma(x \rightarrow \infty) \approx \sqrt{\frac{2 \pi}{x}}\left(\frac{x}{\mathrm{e}}\right)^{x}$

ISSN 2071-0194. Ukr. J. Phys. 2018. Vol. 63, No. 6 to $\Gamma$-functions, whose argument contains $n$, and passing to the limit $n \rightarrow \infty,{ }^{4}$ we obtain the following expression for the change of $J_{+}$when approaching the separatrix:

$2 \pi\left(J_{+0}-J_{+\infty}\right) \approx$

$\approx-a_{+} \Theta_{+}\left[\xi_{+} \ln \xi_{+}-\xi_{+}+\ln \frac{\sqrt{2 \pi}}{\sqrt{\xi_{+}} \Gamma\left(\xi_{+}\right)}\right]$.

The approach to the separatrix and the movement away from it are symmetric in the case of $(+) \rightarrow(-)$ transition. Therefore, Eq. (23), in which the subscripts + are substituted by the subscripts - , is valid for $J_{-\infty}$. Hence, the value of $J_{-}$at a later time moment, when the trajectory is rather far from the separatrix, satisfies the equality

$2 \pi\left(J_{-\infty}-J_{-0}\right) \approx$

$\approx-a_{-} \Theta_{-}\left[\xi_{-} \ln \xi_{-}-\xi_{-}+\ln \frac{\sqrt{2 \pi}}{\sqrt{\xi_{-}} \Gamma\left(\xi_{-}\right)}\right]$.

Now, we should match the processes of approaching the separatrix and moving away from it. We do not know the exact transition time $\tau_{ \pm \mid 0}$. However, we can calculate the difference $\tau_{-\mid 0}-\tau_{+\mid 0}$ and express $\tau_{-\mid 0}$ in terms of the final value for $J_{-}$, which this parameter acquires after the trajectory has moved sufficiently far from the separatrix. Using the pseudocrossing time $\tau_{*}$ as the known reference point, which is independent of $\xi_{i}$, we exclude $\tau_{ \pm \mid 0}$ from the approximate equations

$S_{+}\left(\tau_{+\mid 0}\right) \approx S_{+}\left(\tau_{*}\right)+\Theta_{+}\left(\tau_{+\mid 0}-\tau_{*}\right)$,

$S_{-}\left(\tau_{-\mid 0}\right) \approx S_{-}\left(\tau_{*}\right)+\Theta_{-}\left(\tau_{-\mid 0}-\tau_{*}\right)$

and obtain

$S_{-}\left(\tau_{-\mid 0}\right)-S_{-}\left(\tau_{*}\right) \approx \Theta_{-}\left(\tau_{-\mid 0}-\tau_{+\mid 0}\right)+$

$+\frac{\Theta_{-}}{\Theta_{+}}\left(S_{+}\left(\tau_{+\mid 0}\right)-S_{+}\left(\tau_{*}\right)\right)$.

The expressions for the phase volumes $S_{i}\left(\tau_{i \mid 0}\right)$ can be obtained from $J_{i 0}$ and from the expansions of $I_{i}$ and $u_{i}$ near the separatrix. In their turn, $J_{i 0}$ and $J_{i \infty}$ are related to each other by Eqs. (23) and (24), which ultimately match the states of the system before the approach to the separatrix and after moving away from it. By combining those considerations with the definition of $J_{-*}$, we arrive at the expression

$2 \pi \delta J_{+\rightarrow-}=2 \pi\left(J_{-\infty}-J_{-*}\right)=2 \pi\left(J_{-\infty}-J_{-0}\right)+$

$+\left(2 \pi J_{-0}-S_{-}\left(\tau_{-\mid 0}\right)\right)+\Theta_{-}\left(\tau_{-\mid 0}-\tau_{+\mid 0}\right)-$

$-\frac{\Theta_{-}}{\Theta_{+}}\left(2 \pi J_{+0}-S_{+}\left(\tau_{+\mid 0}\right)\right)+\frac{\Theta_{-}}{\Theta_{+}} 2 \pi\left(J_{+0}-J_{+\infty}\right)$.

It connects the non-adiabatic change of $J_{-}$with the value of $J_{+}$. Furthermore, with the corresponding substitution of subscripts, it is applicable to any transition between the states concerned.

4 Actually, the approach to the separatrix does not take infinite time, but the error of the Stirling approximation is less than $5 \%$ already at $x=2$. 
For the non-linear pendulum, we have $a_{+}=a_{-}=a$ and $a_{t}=2 a$. By substituting Eqs. (23), (24), and (25), as well as the expansion series for $J_{i}$, into Eq. (26) and performing a number of simplifications, we obtain

$2 \pi \delta J_{+\rightarrow-}=-a \Theta_{-} \ln \frac{2 \pi\left(1-\xi_{+}\right)}{\Gamma\left(\xi_{-}\right) \Gamma\left(\xi_{+}\right)}+$

$+\left(1-\xi_{+}\right) b_{[+}^{\prime} \Theta_{-]}+\xi_{i}$-independent terms,

where $b_{i}^{\prime}=b_{i}-a_{i} \ln \left|\Theta_{i}\right|$, and the notation $x_{[i} y_{j}=x_{i} y_{j}-$ $-x_{j} y_{i}$ is used for compactness. The terms that do not contain $\xi_{i}$ are not indicated, because they are irrelevant when calculating the mean-square deviation ${ }^{5}$.

Transition (c) can be considered as a time-inversed version of transition (a), for which $0<\xi_{-}<1$. By introducing $\tilde{P}$, as was done in the main text and putting $\tilde{P} \ll 1$, we obtain

$\ln \Gamma\left(\xi_{-}\right)=\ln \Gamma\left(\xi_{+}\right)-\tilde{P}\left(1-\xi_{+}\right) \ln \xi_{+}+o(\tilde{P})$.

From Eq. (27), keeping the leading-order terms in $\tilde{P}$, we obtain

$2 \pi \delta J_{+\rightarrow-}=-a \Theta_{-} \ln \frac{2 \pi\left(1-\xi_{+}\right)}{\Gamma\left(\xi_{+}\right)^{2}}+$

$+\left(1-\xi_{+}\right) b_{[+}^{\prime} \Theta_{-]}+\xi_{+}$-independent terms.

At sequential transitions across the separatrix, the transition parameters can be considered as uniformly distributed random quantities. The average changes of the adiabatic invariant at transitions (a) and (c) have the opposite signs and mutually compensate each other. However, $J_{ \pm}$, as well as $I_{ \pm}$, undergoes a random fluctuation with the mean-square step

$\left\langle\left(2 \pi\left(\delta J_{+\rightarrow-}-\left\langle\delta J_{+\rightarrow-}\right\rangle\right)\right)^{2}\right\rangle=$

$=M_{4}\left(a \Theta_{-}\right)^{2}+M_{3} a \Theta_{-} b_{[+}^{\prime} \Theta_{-]}+\frac{1}{12}\left(b_{[+}^{\prime} \Theta_{-]}\right)^{2}$,

where

$M_{3}=\int_{0}^{1} d x\left(\ln \frac{1-x}{\Gamma(x)^{2}}-\gamma_{2}\right)\left(x-\frac{1}{2}\right) \approx 0.25$,

$M_{4}=\int_{0}^{1} d x\left(\ln \frac{1-x}{\Gamma(x)^{2}}-\gamma_{2}\right)^{2} \approx 2.6$,

and

$\gamma_{2}=\int_{0}^{1} d x \ln \frac{1-x}{\Gamma(x)^{2}}=-1-\ln 2 \pi$.

Now, let us consider transition (d) and the movement away from the separatrix in the $(t)$ region. Similarly to what was considered above, the system energy at the sequential moments of the maximum approach to the hyperbolic point equal $f_{n}=$ $=f_{0}-n \Theta_{t}$, and the corresponding time moments are connected by the equality

$\tau_{t \mid n+1}=\tau_{t \mid n}+\frac{1}{4} T_{t}\left(f_{n}\right)+\frac{1}{2} T_{t}\left(f_{n}-\Theta_{+}\right)+\frac{1}{4} T_{t}\left(f_{n+1}\right)$,

5 Equation (27) corresponds to Eq. (9) in work [6]. because the slow sections of the $n$-th trajectory loop correspond to the movement away from the hyperbolic point at the energy $f_{n}$, the passage past the hyperbolic point at the energy $f_{n}-\Theta_{+}$ (at the trajectory middlepoint that is close to the separatrix branch between the $(t)$ and $(+)$ regions, the energy of the system decreases by $\left.\Theta_{+}\right)$, and the approach to it at the energy $f_{n+1}$. The energy at the moment of the first maximum approach in the $(t)$ region equals $f_{0}=g_{*}-\Theta_{-}=h_{0}-\Theta_{t}$. However, the trajectory coming from the $(+)$ region can be trapped, only if its energy at the last maximum approach in the $(+)$ region was sufficiently small, i.e. if $h_{0}<\Theta_{t}$; otherwise, the trajectory will transit to the $(-)$ region. Since $h_{0}=\xi_{+} \Theta_{+}$, the transition parameter $\xi_{t}=-f_{0} / \Theta_{t}$ is related to the parameter of the trajectory transition into the $(+)$ region by means of the equality $\Theta_{t}\left(1-\xi_{t}\right)=\Theta_{+} \xi_{+}$, and the transition takes place provided that the condition $0<\xi_{+}<\Theta_{t} / \Theta_{+}$is satisfied. The time required for the trajectory to cross the separatrix equals

$\tau_{t \mid 0}=\tau_{+\mid 0}+\frac{1}{2} T_{+}\left(h_{0}\right)+\frac{1}{2} T_{t}\left(g_{*}\right)+\frac{1}{4} T_{t}\left(f_{0}\right)$

because the trajectory moves away from the hyperbolic point in the $(+)$ region at the energy $h_{0}$, passes past it in the $(t)$ region at the energy $g_{*}$, and, if this energy is insufficient for the trajectory to reach the $(-)$ region, the latter approaches the hyperbolic point again at the energy $f_{0}$. Analogously to the previously considered case, we obtain ${ }^{6}$

$2 \pi\left(J_{t 0}-J_{t \infty}\right)=a_{t} \Theta_{t}\left[\frac{1}{2} \ln \frac{\Gamma\left(\xi_{t}\right) \Gamma\left(\xi_{t}+\frac{\Theta_{+}}{\Theta_{t}}\right)}{2 \pi}+\xi_{t}-\right.$

$\left.-\left(\xi_{t}-\frac{1-\Theta_{+} / \Theta_{t}}{2}\right) \ln \left|\xi_{t}\right|\right]$.

By combining Eqs. (23), (29), and (26), and making the corresponding subscript substitutions, we obtain

$2 \pi \delta J_{+\rightarrow t}=a \Theta_{t} \ln \frac{\Gamma\left(\xi_{t}\right) \Gamma\left(\xi_{t}+\frac{\Theta_{+}}{\Theta_{t}}\right) \Gamma\left(\xi_{+}\right)}{(2 \pi)^{\frac{3}{2}}\left|\xi_{t}-1+\frac{\Theta_{+}}{\Theta_{t}}\right|}+$

$+\frac{\Theta_{t}}{\Theta_{+}} b_{[+}^{\prime} \Theta_{t]} \xi_{t}+\xi_{i}$-independent terms.

Again, putting $\tilde{P} \ll 1$, we find that the transition parameter after crossing the separatrix $\xi_{+}=O(\tilde{P})$, and we may expand $\Gamma\left(\xi_{+}\right)$in a series $\Gamma\left(\xi_{+}\right)=\xi_{+}^{-1}+O(1)$. Furthermore, since $\Theta_{+} / \Theta_{t}=O\left(\tilde{P}^{-1}\right)$, the following expansion is valid:

$\ln \Gamma\left(\xi_{t}+\Theta_{+} / \Theta_{t}\right)=$

$=\ln \Gamma\left(\Theta_{+} / \Theta_{t}\right)+\xi_{t} \ln \left(\Theta_{+} / \Theta_{t}\right)+O(\tilde{P})$.

Then, from Eq. (30), keeping the leading-order terms in $\tilde{P}$, we arrive at

$2 \pi \delta J_{+\rightarrow t}=a \Theta_{t}\left(\ln \frac{\Gamma\left(\xi_{t}\right)}{1-\xi_{t}}+\xi_{t} \ln \frac{\Theta_{+}}{\Theta_{t}}\right)+$

$+\frac{\Theta_{t}}{\Theta_{+}} b_{[+}^{\prime} \Theta_{t]} \xi_{t}+\xi_{i}$-independent terms.

6 This equation corresponds to Eq. (4) in work [6].

ISSN 2071-0194. Ukr. J. Phys. 2018. Vol. 63, No. 6 
In this case, the mean-square step equals

$$
\begin{aligned}
& \left\langle\left(2 \pi\left(\delta J_{+\rightarrow t}-\left\langle\delta J_{+\rightarrow t}\right\rangle\right)\right)^{2}\right\rangle= \\
& =M_{2}\left(a \Theta_{t}\right)^{2}+M_{1} a \Theta_{t}\left(a \Theta_{t} \ln \frac{\Theta_{+}}{\Theta_{t}}+\frac{\Theta_{t}}{\Theta_{+}} b_{[+}^{\prime} \Theta_{t]}\right)+ \\
& +\frac{1}{12}\left(a \Theta_{t} \ln \frac{\Theta_{+}}{\Theta_{t}}+\frac{\Theta_{t}}{\Theta_{+}} b_{[+}^{\prime} \Theta_{t]}\right)^{2},
\end{aligned}
$$

where

$M_{1}=\int_{0}^{1} d x\left(\ln \frac{\Gamma(1-x)}{x}-\gamma_{1}\right)\left(x-\frac{1}{2}\right) \approx 0.001$,

$M_{2}=\int_{0}^{1} d x\left(\ln \frac{\Gamma(1-x)}{x}-\gamma_{1}\right)^{2} \approx 0.77$,

and

$\gamma_{1}=\int_{0}^{1} d x \ln \frac{\Gamma(1-x)}{x}=1+\ln \sqrt{2 \pi}$.

1. J. Nuhrenberg, R. Zille. Quasi-helically symmetric toroidal stellarators. Phys. Lett. A 129, 113 (1988).

2. J. Nuhrenberg, W. Lotz, S. Gori. Theory of fusion plasma. In Proc. Joint Varenna-Lausanne Int. Workshop (Varenna, Italy, 1994) (Editrice Compositori, Bologna, 1994). p. 3.

3. C.D. Beidler, Ya.I. Kolesnichenko, V.S. Marchenko, I.N. Sidorenko, H. Wobig. Stochastic diffusion of energetic ions in optimized stellarators. Phys. Plasmas 8, 2731 (2001).

4. A.V. Timofeev. On the constancy of an adiabatic invariant when the nature of the motion changes. Sov. Phys. JETP 48, 656 (1978).
5. J.R. Cary, D.F. Escande, J.L. Tennyson. Adiabatic-invariant change due to separatrix crossing. Phys. Rev. A 34, 4256 (1986).

6. A.I. Neishtadt. Change of an adiabatic invariant at a separatrix. Sov. J. Plasma Phys. 12, 568 (1986).

7. J.R. Cary, S.G. Shasharina. Helical plasma confinement devices with good confinement properties. Phys. Rev. Lett. 78, 674 (1997).

8. R.G. Littlejohn. Variational principles of guiding centre motion. J. Plasma Phys. 29, 111 (1983).

9. R.G. Littlejohn. Hamiltonian perturbation theory in noncanonical coordinates. J. Math. Phys. 23, 742 (1982).

10. V.I. Arnold. Mathematical Methods of Classical Mechanics (Springer, 1978).

11. T. Andreeva. Vacuum Magnetic Configurations of Wendelstein $7-X$. Preprint IPP III/270 (Max-Planck-Institut für Plasmaphysik, 2002).

Received 29.12.17.

Translated from Ukrainian by O.I. Voitenko

A.B. Тихиนั

\section{СТОХАСТИЧНА ДИФУЗІЯ ЕНЕРГІЙНИХ}

ЙОНІВ У СТЕЛАРАТОРАХ ТИПУ WENDELSTEIN

$\mathrm{P}$ е $з$ ю м е

Розглянуто беззіткненнєву стохастичну дифузію енергійних йонів в оптимізованих стелараторах типу Wendelstein, існування якої в цих пристроях було передбачено раніше за допомогою спрощеної теорії переходу іонів через сепаратрису. Обчислено стрибки адіабатичного інваріанта у стелараторних магнітних конфігураціях. Аналіз отриманих виразів підтверджує важливість стохастичної дифузії та демонструє, що коефіцієнт дифузії може значно перевищувати відомі оцінки. 